\title{
An att site-based recombination reporter system for genome engineering and synthetic DNA assembly
}

\author{
Michael J. Bland ${ }^{1,2}$, Magaly Ducos-Galand ${ }^{1,2}$, Marie-Eve Val ${ }^{1,2}$ and Didier Mazel ${ }^{1,2^{*}}$ (D)
}

\begin{abstract}
Background: Direct manipulation of the genome is a widespread technique for genetic studies and synthetic biology applications. The tyrosine and serine site-specific recombination systems of bacteriophages HKO22 and ФC31 are widely used for stable directional exchange and relocation of DNA sequences, making them valuable tools in these contexts. We have developed site-specific recombination tools that allow the direct selection of recombination events by embedding the attB site from each system within the $\beta$-lactamase resistance coding sequence (bla).

Results: The HK and ФC31 tools were developed by placing the attB sites from each system into the signal peptide cleavage site coding sequence of bla. All possible open reading frames (ORFs) were inserted and tested for recombination efficiency and bla activity. Efficient recombination was observed for all tested ORFs (3 for HK, 6 for $\Phi(31)$ as shown through a cointegrate formation assay. The bla gene with the embedded attB site was functional for eight of the nine constructs tested.

Conclusions: The HK/DC31 att-bla system offers a simple way to directly select recombination events, thus enhancing the use of site-specific recombination systems for carrying out precise, large-scale DNA manipulation, and adding useful tools to the genetics toolbox. We further show the power and flexibility of bla to be used as a reporter for recombination.
\end{abstract}

Keywords: Site-specific recombination, Tyrosine recombinase, Serine recombinase, Genetic engineering

\section{Background}

The ability to precisely and directly manipulate DNA is important for functional studies and the synthetic assembly of large genetic constructs. Site-specific recombinase (SSR) systems are widely used as tools to rearrange, insert, remove, and join DNA with virtually no upper limit in size. For biotechnology purposes, this can include the insertion of exogenous DNA into chromosomes, the fusing of DNA molecules, or the construction of synthetic gene networks [1]. The tyrosine (Y-rec) and serine (S-rec) recombination families are named for the catalytic residue of their respective integrase (Int) protein. Important members of the Y-rec family include the $\lambda$-like phage recombination systems,

\footnotetext{
* Correspondence: mazel@pasteur.fr

'Unité Plasticité du Génome Bactérien, Département Génomes et Génétique, Institut Pasteur, 75015 Paris, France

2UMR3525, Centre National de la Recherche Scientifique, 75015 Paris, France
}

which include $\lambda$ and the closely related phage HK022 (hereafter referred to as HK). The ФC31 recombinase system is an important member of the S-rec family [2]. Both HK and $\Phi C 31$ systems comprise attB/attP attachment sites that serve as points of recombination, and the recombinases that catalyze recombination. In each family, DNA exchange requires host-encoded proteins for recombination that differ between systems. These systems are attractive due to their directionality and stability, and both systems are functional in prokaryotic and eukaryotic organisms [3-5].

Mechanistically, attB and attP integrative recombination forms attL and $a t t R$ sites. The reverse $a t t L \times a t t R$ excisive reaction also requires Int as well as a recombination directionality factor (RDF), named Xis in the HK system and gp3 in the ФC31 system [6], typically supplied in trans from a helper plasmid, a non-replicating DNA molecule, or as mRNA [7]. Structurally, HK and 
ФC31 att sites differ in size, with the HK attB sites being generally shorter than the HK attP sites, 21 base pairs (bp) vs 234 bp [8, 9]; in addition, attP contains binding sites for Int and Xis along with host-encoded proteins Fis and IHF [8-11]. ФC31 attB and attP sites are similar in size $(\sim 50 \mathrm{bp})$ and do not require additional proteins to carry out recombination [12].

The use of SSRs generally involves selecting the recombination event through the use of a marker gene within the inserted sequence whose presence or absence would indicate successful integration [1]. Genes can be activated following recombination through either removal of blocking DNA sequences or by bringing together physically separated congruous sequences, with the recombination site embedded within the gene or between the promoter and coding sequence. This approach has long been used with the popular CRE/loxP [13] and Flp/FRT [14] systems. The $\beta$-lactamase (bla) gene is an attractive marker, as it is a useful reporter gene for both pro- and eukaryotic applications [15]. Protein chimeras of $\beta$-lactamase demonstrate tolerance to exogenous peptide insertions [16], even for domains of unknown function [17]. A split gene reassembly approach using bla has also been developed to discover directed evolutionmodified SSR enzymes capable of recombining designer sequences [18]. The bla signal peptide is an attractive region for peptide insertion [19], as insertions between the signal peptide sequence and the rest of the coding gene have minimal interference with protein function [20]. As we wished to expand the available molecular toolbox, we created a set of recombination reporters consisting of the attB of $\mathrm{HK}$ and ФC31 inserted in frame with bla, allowing expression of the gene and enabling the direct selection of recombination events. The selective agent is not expressed when the att sites are in attL and attR form, as the reporter gene fragments are physically separated (Fig. 1a).

This approach has been used to explore the physical structure of the $E$. coli genome [21, 22]. Genome engineering of the two Vibrio cholerae chromosomes used this tool to understand the evolutionary and genetic implications of multi-chromosomal bacteria [23]. We have used HK recombination in tandem with the $\lambda$-lacZ system from [21] to exchange DNA between the two V. cholerae chromosomes in a recombination-mediated cassette exchange (RMCE), resulting in large-scale chromosomal rearrangements [23]. Because the lac $Z$ reporter allows the observation of recombination events but not to select for them, we developed a reporter system for HK recombination based on antibiotic selection. We have used an $\mathrm{HK}$ attB site placed in-frame within the $\beta$-lactamase (bla) gene to carry out relocation of the S10-spec- $\alpha$ ribosomal locus in $V$. cholerae in order to study the consequences of essential gene positioning as it relates to dosage [24]. We further used HK-bla to carry out large-scale genome inversions around the origin region (ori) of V. cholerae chromosome one (Chr1) to shift the timing of the initiation of chromosome two (Chr2) replication relative to Chr1 in order to study the mechanisms involved in bacterial chromosome replication timing [25].

Here, we describe the construction and validation of HK-bla and a similar tool using the serine ФC31 att system (ФC31-bla). We placed attB sites from each system immediately downstream of the bla signal peptide coding sequence, which directs transport of $\beta$-lactamase to the periplasm and is removed in the mature protein. $\beta$-lactamase is generally tolerant of insertions into this region. When each system is present as attL and attR sites, they are associated with fragment sequences bla' (the $5^{\prime}$ region upstream of the cleavage site including the promoter and signal sequence) and 'bla (the 3 ' region comprising the mature protein sequence), respectively (Fig. 1a). In addition, the cognate att site partners show high recombination frequencies without the presence of blaresistant background from the fragmented bla gene. These systems are extremely useful due to their ability to directly select for recombination through resistance to $\beta$-lactam antibiotics. They also have the potential to be used within synthetic biology frameworks for constructing and precisely inserting large genetic assemblies, making them useful additions to the molecular biology toolbox for both synthetic and molecular applications.

\section{Results}

In-frame insertion of att $_{\mathrm{HK}}$ sites within the B-lactamase gene The $\beta$-lactamase gene has a 23 -amino acid (aa) signal peptide sequence for protein transmembrane transport that is cleaved during protein maturation [26]. We inserted the attB sequences in frame into the junction between the encoded signal sequence and the mature protein (Fig. 1a), as this region is tolerant to sequence insertions [19]. To avoid interfering with the $\beta$-lactamase coding sequence we took into account attB length and the amino acid sequence of the translated att sequence, so as to avoid frameshift or stop codon insertion.

Recombination frequency in $a t t B_{\mathrm{HK}}$ sites decreases with size The $a t t B_{\mathrm{HK}}$ site comprises a 7 bp core, or overlap, $(\mathrm{O})$ region where strand exchange occurs, and flanking $B$ and $\mathrm{B}^{\prime}$ arm regions of $7 \mathrm{bp}$ each that are recognized by Int monomers to form a synaptic complex, although sites shorter than this $21 \mathrm{bp}$ have been shown to be functional but with low efficiency [27]. To allow recombination, the $\mathrm{O}$ region between attB and attP must 

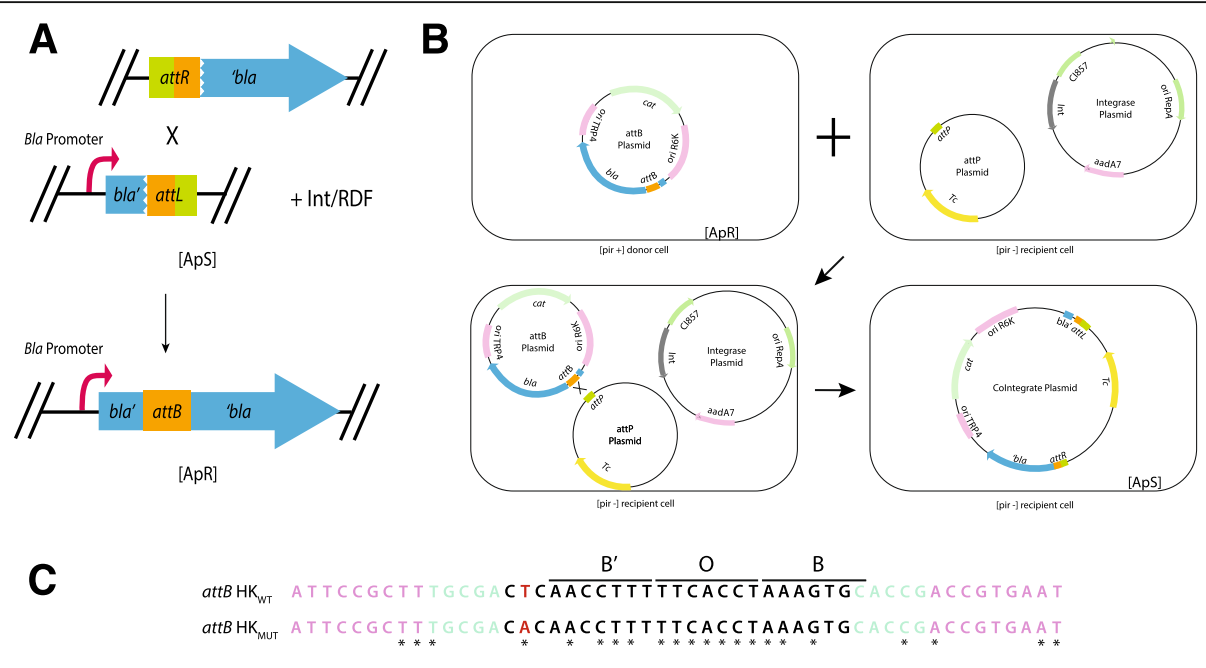

D

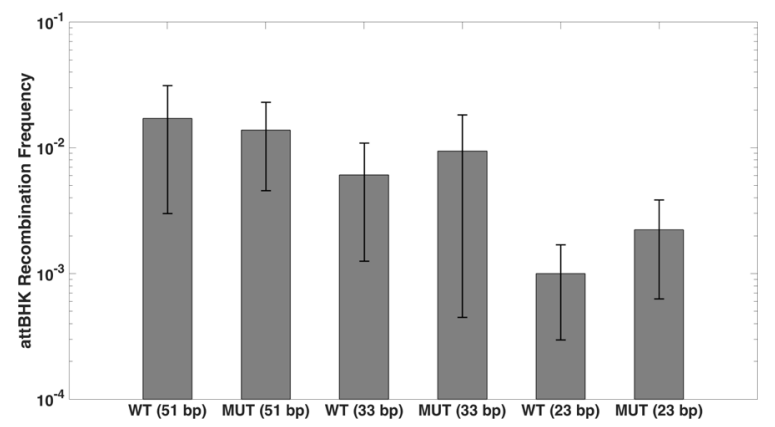

Fig. 1 Schematic representation of attB-bla system and the conjugative assay used to test att sites. a In the selective tool, the bla gene is fragmented such that the $5^{\prime}$ promoter and signal sequence are associated with an attL site, and the partner attR is associated with the $3^{\prime}$ region. Each component is placed at separate loci, either on the genome or a plasmid, depending on the application. $\mathbf{b}$ Conjugation of the attB plasmid into a recipient strain containing the attP and integrase plasmids to form the att $R$ and attL partners with bla gene fragments. c Sequence of the HKO22 attB site. We tested $a t t B_{\mathrm{HK}}$ sites of three different lengths to avoid potential interference with bla function and protein export, $51 \mathrm{bp}$ (violet), $33 \mathrm{bp}$ (teal), and $23 \mathrm{bp}$ (black). To increase the number of potential open reading frames, we introduced a $T \rightarrow$ A nucleotide change into the $a t t B$ sequence, indicated in red. The $\mathrm{BOB}^{\prime}$ core region is demonstrated by black lines. Stars indicate bases in common with attP $P_{\mathrm{HK}}$. Recombination points flank the core $\mathrm{O}$ region. $\mathbf{d}$ Recombination results of att $B_{H K}$ sequences. These six sequences were tested using a plasmid conjugation assay in a context independent of the bla gene [29]. This demonstrated that the introduced mutation did not interfere with recombination efficiency and the length of the attB site had a negative correlation with recombination frequency. As we wished to use a shorter sequence to avoid interfering with bla functionality following attB site insertion, we based our subsequent ORF constructions on the 23 bp mut form, despite the fact that it recombines at a lower frequency than the 51 and 33 bp wt sequences

perfectly overlap, and the arm regions must share similarity. Flanking the core minimal region, there are homologous nucleotides that may play an additional role in recombination efficiency [10, 28]. Insertion of attB into bla extends the gene and could affect either transport through the membrane or mature enzyme function. It is therefore necessary to test different open reading frames encoded by the $a t t B_{\mathrm{HK}}$ sequence to avoid unwanted interference with bla. The native att $B_{\mathrm{HK}}$ sequence encodes two open reading frames (ORFs) that do not have stop codons. As we wished to increase the potential sequences we could test within bla, we added a third potential ORF by mutating one bp just outside of the B' region (Fig. 1c; Fig. 2a) [8, 27]. We compared these "mutant" attB sites to the "wild-type" sites to ensure there was no loss of recombination frequency (Fig. 1d).
The 23, 33, and 51 bp "wild type" and "mutant" att $B_{\mathrm{HK}}$ sequences were tested by placing them on the conditionally replicating conjugative plasmid pSW23T containing an ori $_{\mathrm{RP} 4}$ for plasmid conjugation and ori $V_{\mathrm{R} 6 \mathrm{~K} \gamma}$ for $\pi$ protein replication dependence (Fig. 1b); [29]. As these plasmids do not replicate in bacterial strains not expressing the $\pi$ protein, conjugation into non- $\pi$ expressing DH5 $\alpha$ leads to plasmid loss unless att recombination occurs. The DH5 $\alpha$ recipient strain houses plasmid pHK11 $\Delta \mathrm{amp}$, which has the $a t t P_{\mathrm{HK}}$ partner site, and pHK-Int, which expresses the HK integrase under control of the temperature-dependent CI857 promoter [30]. Following conjugation, recombination frequency was calculated by measuring the ratio of recovered colonies (representing co-integrates) over the number of recipient colonies [31]. Recombination frequencies were 


\section{A HK attB Open Reading Frames}

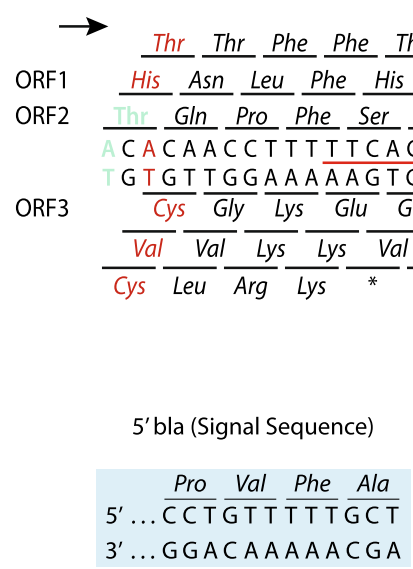

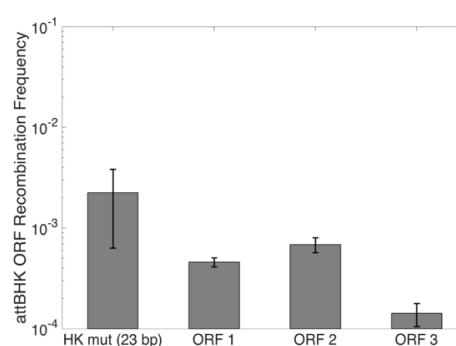

ORF1 $\frac{\text { His }}{\text { C AC A A C C C T T T T T }} \frac{\text { Leu }}{\text { CAC Che }} \frac{\text { Leu }}{\text { CTA A A G }} \frac{\text { Trp }}{\text { TGG }}$ ORF2

Thr Gln Pro Phe Ser Pro Lys Val $\frac{T h r}{\text { ACA }} \frac{\text { Gln }}{\text { CAA }} \frac{\text { Pro }}{\text { CCT }} \frac{\text { Phe }}{\text { T T T T CA }} \frac{\text { Pro }}{\text { CCT }} \frac{\text { Lys }}{\text { A A A G T G }}$ ORF3 3'bla (Mature $\beta$-lactamase)

His Pro Glu Thr $\overline{\mathrm{CAC}} \overline{\mathrm{CCA}} \overline{\mathrm{GAA}} \overline{\mathrm{ACG}} \ldots 3^{\prime}$ GTGGGTCT T T GC ... 5'

Ala Thr Leu Gly Glu Lys Gly Cys GCC ACT TTA GGT GAA AAAGGT $\frac{\text { TGT }}{\text { TA }}$

\section{B ФC31 attB Open Reading Frames}
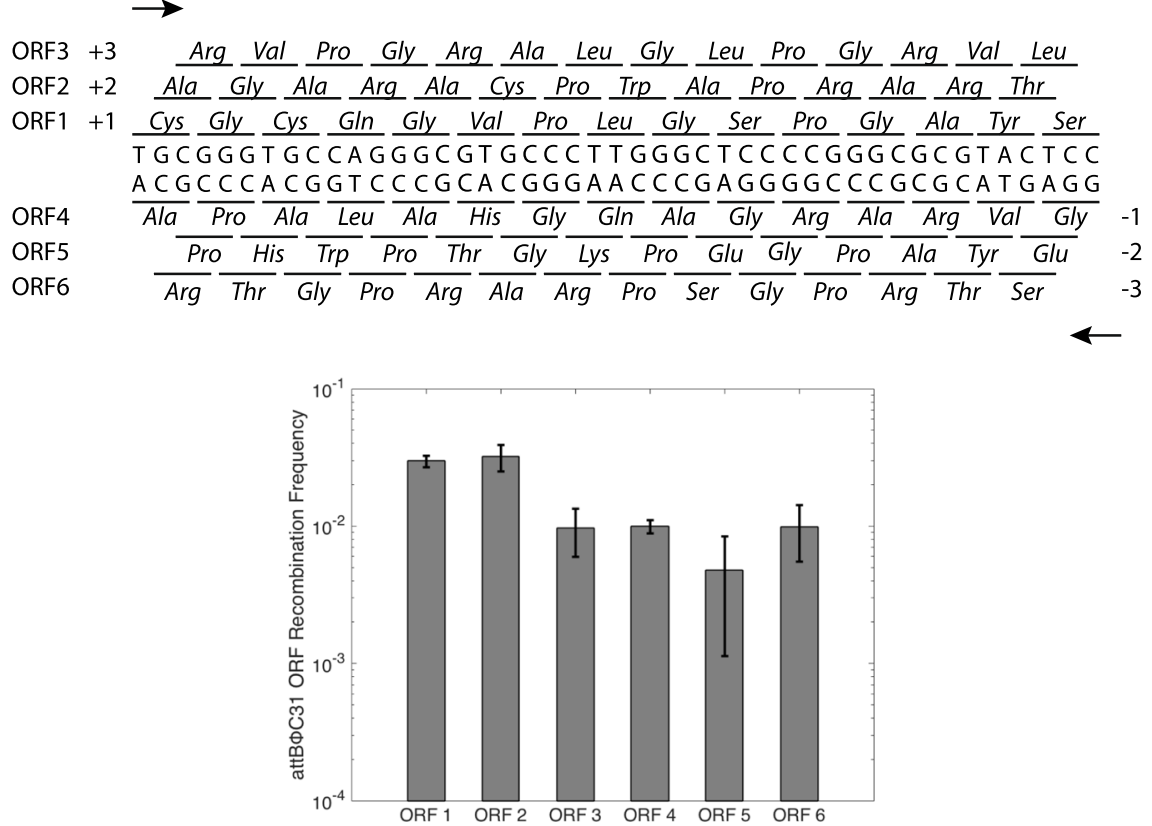

Fig. 2 Sequences and recombination frequencies of HK and DC31 attB sites. The three ORFs for HK and the six ORFs for $\Phi$ C31 were inserted into bla and tested using the conjugation assay as in Fig. 1a. The six open reading frames of the 23 bp attB HK site are shown. As in Fig. 1, black nucleotides represent the $23 \mathrm{bp} \mathrm{HK}$ sequence, with the corresponding amino acids also in black. The red nucleotide shows the base changed from the original attB, with the resulting amino acid changes also shown in red. Nucleotides and amino acids in teal represent sequences flanking the 23 bp site. Horizontal arrows indicate the direction of transcription, and asterisks indicate a stop codon. For both $\mathbf{a}$ and $\mathbf{b}$, the sequence of recombination exchange is indicated by a horizontal red line. As described in the text, three open reading frames did not have a stop codon and were able to be tested for bla insertion. The recombination frequencies of these open reading frames compared to the $23 \mathrm{bp} H \mathrm{~K}$ attB site are shown in the bar graph. The open reading frames are also shown in context of the bla sequence flanking the insertion site. Note that to keep the attB site in frame with bla, nucleotides were added to either the $5^{\prime}$ or $3^{\prime}$ end of the site, which changed the expected amino acid residue for ORFs 1 and 3 compared to the original attB. The background colors highlighting the sequence correspond to Fig. 1a. The recombination frequencies of the different ORFs were compared using 1-way ANOVA followed by a Tukey-Kramer test. Each of the HK ORF recombination frequencies are significantly different ( $p<0.05)$. $\mathbf{b}$. None of the six $\Phi$ C31 ORFs encode a stop codon. ORFs 1 and 2 recombine at a higher rate than ORFs 3-6 ( $p<0.001)$

similar between the different sites, with only a 10-fold reduction in recombination observed for the $23 \mathrm{bp}$ sites compared to the larger attB sites (Fig. 1c). As we wished to use a shorter sequence to avoid interfering with bla functionality following attB site insertion, we based our subsequent tests on the $23 \mathrm{bp} a t t B_{\mathrm{HK}}$ mutant form. 
Placing a single nucleotide mutation in the $23 \mathrm{bp}$ $a t t B_{\mathrm{HK}}$ site enables the use of three ORFs that would potentially allow bla function following their insertion into the gene (Fig. 2a). These ORFs were inserted separately into bla downstream of the signal sequence and cloned into pSW23T in a $\pi+$ host. Following construction of these plasmids, we measured the ampicillin minimum inhibitory concentration (MIC) of each to test and measure bla function. All ORFs provided resistance to ampicillin at an MIC $>256 \mu \mathrm{g} / \mathrm{ml}$ (Table 1). Recombination frequencies were then tested using the conjugation assay as above. The three HK ORF constructions demonstrated a wide range of recombination efficiencies, with the ORF 2 construct recombining at the highest level, and the ORF 3 construct recombining at the lowest (Fig. 2a). Thus, we used ORF 2 for the final construction of this tool.

ФC31 attB $\times$ attP recombination is functional in all six ORFs We designed $a t t B_{\Phi C 31}$ sites for all six possible ORFs maintaining at least the minimal sequence necessary for recombination [32] and inserted them into bla. Ampicillin resistance and recombination frequency were determined as with the HK system. Five of six ORFs were found to provide MICs greater than $256 \mu \mathrm{g} / \mathrm{ml}$, with the ORF 5 construction being the only sequence to interfere with $\beta$-lactamase function (MIC $=6 \mu \mathrm{g} / \mathrm{ml}$ - Table 1 ). ФC31 pSW23T-bla plasmids were conjugated into a DH5 $\alpha$ strain harboring plasmids pФC31-Int and pФC31-attP. All six ORFs were able to recombine successfully, with ORF constructions 1 and 2 recombining at a higher rate, on the order of $10^{-2}$, than ORFs 3-6, which recombined at an average rate of $10^{-3}$ (Fig. 2b). We found this difference to be significant using a 1-way ANOVA $(p<0.001)$ followed by a post-hoc TukeyKramer test $(p<0.001)$. Additionally, all six ФC31 ORF constructions recombined at a higher rate than $\mathrm{HK}$ ORFs 1-3 (Fig. 2).

Table 1 Minimum inhibitory concentration (MIC) of att $B_{H K}$ and attB $_{\Phi C 31}$ ORFs inserted into $\beta$-lactamase

\begin{tabular}{ll}
\hline Ampicillin Resistance of bla-attB ORFs & MIC $(\mu \mathrm{g} / \mathrm{ml})$ \\
\hline HK022 ORF1 & $>256$ \\
HK022 ORF2 & $>256$ \\
HK022 ORF3 & $>256$ \\
ФC31 ORF1 & $>256$ \\
ФC31 ORF2 & $>256$ \\
ФC31 ORF3 & $>256$ \\
ФC31 ORF4 & $>256$ \\
DC31 ORF5 & 6 \\
ФC31 ORF6 & $>256$ \\
\hline
\end{tabular}

\section{Discussion}

In this study, we describe the construction of two sitespecific recombination tools useful for DNA manipulation applications. The utility of this attB-bla tool is based on its incorporation of the widely used HK and ФC31 recombination systems. In the case of HK, the removal of sequences flanking the BOB' core region reduced $a t t B \times$ attP recombination. This reduction could be due to the removal of bases outside of the attB core that have homology with the attP sequence, which may act to stabilize the attB/attP complex. However, obtaining the highest possible recombination frequency was not critical for the design of this system, as our main concern was $\beta$-lactamase function following insertion of the att sites into the bla coding frame.

In directly comparing the two systems, the $\Phi C 31$ site appears to recombine at a similar frequency to the $51 \mathrm{bp}$ HK sites and the 23 bp HK ORFs incorporated into bla have a lower recombination frequency (Fig. 2). This decrease is likely due to the reduction of size of the $a t t B_{\mathrm{HK}}$ site, as the recombination frequencies for the smaller HK site tested independently of bla insertion are not different from the frequencies obtained when they are embedded in bla (Fig. 1). Reported differences between recombination systems in the literature may result from differences in protocols and practices. A recent review of $\Phi C 31$ found a wide range of reported recombination frequencies for this recombinase [33]. To our knowledge, the only information comparing HK and ФC31 recombination frequencies reports $\mathrm{HK}$ recombining at a higher frequency than $\Phi C 31$ [34]. However, this study used a clonetegration technique where constructs were recombined into native att sites on either the $E$. coli chromosome for $a t t P_{\mathrm{HK}}$ or Salmonella typhimurium for att $P_{\Phi \mathrm{C} 31}$.

While testing bla expression with inserted ORFs, we observed that ФC31 ORF 5 interfered with bla expression, while ФC31 recombination was not affected (Table 1, Fig. 2b). The bla gene used for our system originates from pBR322 and belongs to the TEM-1 class of $\beta$-lactamases. The signal sequence is recognized by the Sec export pathway that transports unfolded proteins across the cytoplasmic membrane [26, 35]. DNA secondary structures could be a source of transcription interference, as ORF 5 forms a 30 bp hairpin $(\Delta G$ at $37{ }^{\circ} \mathrm{C}=-9.09 \mathrm{kcal} / \mathrm{mol}$ ). However, hairpins are formed in all 6 ORFs at similar $\Delta \mathrm{G}$, making it unlikely that this factor alone prevents bla expression. At the translation level, the overall charge of the first 5 amino acids following the signal sequence can influence cleavage and cross-membrane transport, as they generally have an overall negative charge [36]. For ORF 5, the overall negative charge of this region is +2 . Again, however, this is unlikely to explain the loss of bla expression, as only ORF 1 has an overall negative charge, at -1 . The 
amino acids in the 1 and 2 position after the cleavage site can also influence protein function [37, 38]. For ORF 5, the first two amino acids are glycine and serine. Analysis of 307 proteins from the SPdb database [39] found that in Gram-negative bacteria, glycine occurs in the 1st position in $6.19 \%$ of proteins, and serine appears in the 2nd position in $5.54 \%$ of proteins. [40]. Additionally, two of the 307 Gram-negative proteins analyzed in this study begin with glycine-serine. Thus it is unlikely that the first two residues of the ORF 5 sequence alone interfere with protein transport. More experimental and analytical work is needed to determine the source of bla expression interference.

The high tolerance of bla to in-frame DNA sequence insertion downstream of the bla promoter and leader peptide sequence allows for further modifications of this system through insertion of potentially large ORFs. This approach has already been proposed as an "ORF-trap" to capture DNA encoding protein fragments [41]. Indeed, large ORFs in frame with bla may not greatly reduce $\beta$ lactamase function, although export to the periplasm can be inhibited [42]. Additionally, as attB and attP site reactivity can be modified through mutations to their respective core sequences, variable non-reacting "synthetic" att sites can be designed for sequential introduction into the bacterial chromosome [43].

Integration of exogenous DNA sequences into genomes by SSRs generally involves the recombination of an attP site on the inserted sequence with an endogenous chromosomal attB or pseudo-attB site [1]. The use of genome editing technologies allows the insertion of recombination sites that differ from native sites in location and sequence. Native att sites may be located in undesirable regions of the genome, for example, in an active gene locus, or a locus subject to silencing. Additionally, dosing effects can be observed in bacterial species dependent on a gene's location in the chromosome [24]. Engineering att site recognition by Int proteins allows the creation of semi-synthetic partner sites [27, 43]. This would avoid recombination with other native att sites, and could allow rapid construction of synthetic gene networks. The addition of FRT sites flanking the bla-attB cassette would further allow for removal of the resistance selection marker gene. Similarly, gene-editing technologies could allow the targeted insertion of att sites to serve as landing pads for insertion. In this way, the bla'-attL sequence from our system can be inserted into a genome, into which a sequence containing the partner attR-'bla can be inserted through attL $\mathrm{x}$ attR recombination. This framework has already been proposed for the construction and insertion of metabolic networks into eukaryotic cell lines [44]. Our system adds the advantage of avoiding marker expression until recombination, making it versatile for synthetic applications as well as genome-scale engineering.

\section{Conclusions}

We describe here the construction of new tools based on two different site-specific recombination systems, the tyrosine recombinase HK, and the serine recombinase $\Phi C 31$. Recombination for each system is reported based on the reconstitution of the bla ampicillin resistance gene, providing resistance to $\beta$-lactam antibiotics as a selective agent. Both HK-bla and ФC31-bla are useful for selecting recombination events in a genomic context due to a high rate of recombination frequency, directionality based on the recombination proteins supplied in trans, and the ability to carry out in vivo genomic rearrangements. We have previously used this tool in our lab to carry out largescale reorganization of the $V$. cholerae chromosomes to study the importance of chromosome size in multichromosomal bacteria [23], the relevance of genome position and chromosome location for gene dosage and its evolutionary importance [24], and the timing of $V$. cholerae chromosome replication [25]. The importance of these tools lie in their capacity to exist simultaneously in the cell at two separate loci without expression of the marker gene until expression of the recombination proteins is induced.

\section{Methods \\ Bacterial strains and media}

Bacterial strains used in this study are described in Table 2. All strains were grown in lysogeny broth (LB) medium at $30^{\circ} \mathrm{C}, 37^{\circ} \mathrm{C}$, or $42^{\circ} \mathrm{C}$ depending on plasmid temperature-sensitivity. Antibiotic and nutritional supplement concentrations were as follows: ampicillin (Ap):

Table 2 Bacterial strains used in this study

\begin{tabular}{|c|c|c|}
\hline \multicolumn{3}{|l|}{ E. coli } \\
\hline \multicolumn{3}{|r|}{ Reference/Source } \\
\hline$\beta 2163$ & $\left(F^{-}\right)$RP4-2-Tc::Mu $\Delta$ dapA::(erm-pir) $\left[\mathrm{Km}^{\mathrm{R}} \mathrm{Em}^{\mathrm{R}}\right]$ & [29] \\
\hline$\pi 1$ & DH5a $\Delta$ thyA::(erm-pir116) $\left[\mathrm{Em}^{\mathrm{R}}\right]$ & [29] \\
\hline MFDpir & 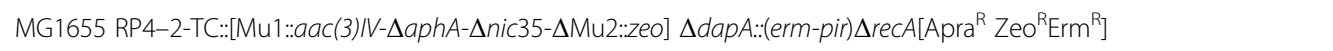 & [47] \\
\hline PGB-8557 & DH5a strain containing plasmids pHK $\Delta-A m p$ and pHK-Int $\left[c^{R} S p^{R}\right]$ & this study \\
\hline PGB-E274 & DH5a strain containing plasmids $p \Phi C 31$-attP and $p \Phi C 31-\operatorname{lnt}\left[T c^{R} S p^{R}\right]$ & this study \\
\hline $\begin{array}{l}\text { One Shot }{ }^{\circledR} \\
\text { Top10 }\end{array}$ & F- mcrA $\Delta$ (mrr-hsdRMS-mcrBC) $\Phi 80 / a c Z \Delta M 15 \Delta$ lacX74 recA1 araD139 $\Delta$ (araleu)7697 gall galK rpsL (StrR) endA1 nupG & $\begin{array}{l}\text { ThermoFisher } \\
\text { Scientific }\end{array}$ \\
\hline
\end{tabular}


$100 \mu \mathrm{g} / \mathrm{ml}$, carbenicillin (Carb): $100 \mu \mathrm{g} / \mathrm{ml}$, kanamycin (Km): $25 \mu \mathrm{g} / \mathrm{ml}$, chloramphenicol, (Cm): $25 \mu \mathrm{g} / \mathrm{ml}$, tetracyclin (Tc): $15 \mu \mathrm{g} / \mathrm{ml}$, spectinomycin (Sp): $100 \mu \mathrm{g} / \mathrm{ml}$, erythromycin (Em): $20 \mu \mathrm{g} / \mathrm{ml}$, with nutritional supplements diaminopimelic acid (DAP): $300 \mu \mathrm{M}$, and thymine (dT): $300 \mu \mathrm{M}$.

\section{Cloning}

Basic cloning steps were performed using the following tools and appropriate protocols: for DNA purification, a QIAquick PCR purification kit (QIAGEN) was used. Plasmid minipreps were performed using the GeneJET Plasmid Miniprep kit (Life Technologies). All PCR reactions for plasmid construction were performed using the Phusion High-Fidelity PCR Master Mix (Life Technologies), and all diagnostic PCR reactions were performed using DreamTaq DNA Polymerase (Life Technologies). Oligonucleotides were synthesized by Sigma-Aldrich and Eurofins Genomics. Oligonucleotides were phosphorylated by $\mathrm{T} 4$ polynucleotide kinase (NEB). DNA was sequenced by GATC Biotech and Eurofins Genomics.

\section{Construction of plasmids}

Insertion of attB sequences into pSW23T was performed by annealing phosphorylated oligos containing the respective att sequence with overhangs overlapping with $\mathrm{BamHI}$ and EcoRI restriction sites, followed by cloning of these sequences into the pSW23T fragment. Insertion of attP sequences into pHK11-Amp was similarly performed. The various attB ORFs for both $\mathrm{HK}$ and $\Phi C 31$ were inserted into the $\beta$-lactamase (bla) by overlapping PCR, in which the $5^{\prime}$ region of bla was amplified from pMP58 using oligos MV26 and the appropriate reverse attB oligo, and the 3' bla region amplified using a forward attB oligo and JB13. These products were gel purified and co-amplified using oligos MV26 - JB13 to form a DNA fragment containing $b l a$ with the inserted $a t t B$. This product was digested with EagI and EcoRI and cloned into pSW23T and transformed into MFDpir. The pMP58 bla gene comes from pUC19.

To make plasmid pPhiC31-Int, we first deleted the Xbal site in pZJ7 (a kind gift of Jia Zhao and Sean Colloms) by digestion with SpeI-XbaI followed by religation to make plasmid pZJ7 $\Delta$ XbaI. The $\Phi C 31$

Table 3 Plasmids used in this study

\begin{tabular}{|c|c|c|}
\hline Name & Description & Reference/Source \\
\hline pSW23T & pSW23::oriTRP4; $\left[\mathrm{Cm}^{\mathrm{R}}\right]$; oriVR6K & [29] \\
\hline pSU38 & orip15A $\left[\mathrm{Km}^{\mathrm{R}}\right]$ & [48] \\
\hline pHK-Int & 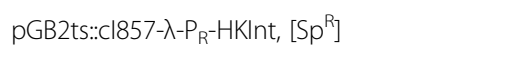 & [30] \\
\hline pHK11-Amp & pLDR1 1::attP_HK, $\left[A p^{R}, T c^{R}\right]$ & [30] \\
\hline pSC101 & pSC101ts, repA $\left[c^{R}\right]$ & [49] \\
\hline pUC19 & oriColE1, lacZa $\left[\mathrm{Ap}^{\mathrm{R}}\right]$ & [50] \\
\hline pBAD43 & oripSC101, PBAD::MCS,[Sp $\left.{ }^{R}\right]$ & [51] \\
\hline $\mathrm{pHK} 11 \Delta \mathrm{amp}$ & pHK11-Amp::attP_HK, $\Delta$ Amp, $\left[T c^{R}\right]$ & this study \\
\hline pMP96 & 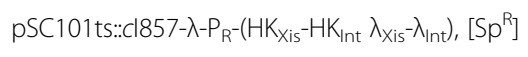 & [23] \\
\hline pMP58 & pSC101ts::oriTRP4;repA, $\left[\mathrm{Cm}^{\mathrm{R}}, \mathrm{Ap}^{\mathrm{R}}\right]$ & this study \\
\hline pMDG1 & pMP58;bla::attB_HK, $\left[\mathrm{Ap}^{\mathrm{R}}, \mathrm{Cm}^{\mathrm{R}}\right]$ & this study \\
\hline pMDG2 & pSW23T::bla::attB_HK from pMDG1 & this study \\
\hline pMDG3 & a/pSU38::attR_HK, $\left[\mathrm{Ap}^{\mathrm{R}}\right]$ & this study \\
\hline pMDG4 & pSW23T::attL_HK, $\left[\mathrm{Cm}^{\mathrm{R}}\right]$ & this study \\
\hline pMJM1 & pSW23T::attB_HKwt, $\left[\mathrm{Cm}^{\mathrm{R}}\right]$ & this study \\
\hline pMJM2 & pSW23T::attL_HKmut, $\left[\mathrm{Cm}^{\mathrm{R}}\right]$ & this study \\
\hline pMJM3 & pSW23T::attL_HK40, $\left[\mathrm{Cm}^{\mathrm{R}}\right]$ & this study \\
\hline pMJM4 & pSW23T::attL_HK30, $\left[\mathrm{Cm}^{R}\right]$ & this study \\
\hline pJB6 & pSU38 $\Delta$ :attR_HK-attL_ $\lambda,\left[\mathrm{Ap}^{\mathrm{R}}\right]$ & this study \\
\hline pJB7 & pSW23T::attR_HK-attL_$\lambda,\left[\mathrm{Cm}^{\mathrm{R}}\right]$ & this study \\
\hline pJB8 & pBAD43::HK xis $_{\text {is }}-H K_{\text {Int }} \lambda_{x_{i s}}-\lambda_{\text {Int }}\left[\mathrm{Sp}^{\mathrm{R}}\right]$ & this study \\
\hline pZJ7 & pBAD33::ФC31lnt, $\left[\mathrm{Cm}^{\mathrm{R}}\right]$ & J. Zhao and S. Colloms \\
\hline $\mathrm{pZJJ} \Delta \mathrm{Xbal}$ & pZJ7 with Spel - Xbal fragment deleted & this study \\
\hline pPhic31-Int & 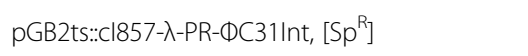 & this study \\
\hline pPhiC31-attP & pHK11 1 amp::attP_ФC31, $\left[T c^{R}\right]$ & this study \\
\hline
\end{tabular}


integrase gene was amplified using oligos PhiC31 IntF and PhiC31 IntR. The pHK-Int backbone was amplified using oligos JB485 and JB486. These oligos produce DNA fragments with overlapping ends, which were then joined by Gibson assembly [45]. Plasmids used in this study are listed in Table 3 and oligonucleotides in Table 4.

\section{Recombination assay}

Recombination frequencies were tested by performing a conjugation assay in which the plasmid pSW23T containing the ori $T_{\mathrm{RP} 4}$ transfer region and ori $V_{\mathrm{R} 6 \mathrm{~K}} \pi-$ controlled replication origin were transferred from the $\pi+/$ DAP- donor strain MFDpir to a recipient strain

Table 4 Oligonucleotides used in this study

\begin{tabular}{|c|c|}
\hline Oligonucleotide & Sequence $5^{\prime}-3^{\prime}$ \\
\hline PhiC31 Int F & ATGTACTAATCTAGAGAAGAGGATCAGAAATGGACACGTACGCGGGTGC \\
\hline PhiC31 Int R & CAAGCTTGCATGCCTGCAGG \\
\hline JB13 & AGCGGGTGTTCCTTCTTCACTG \\
\hline JB485 & TCTTCTCTAGATTAGTACATGCAACCA \\
\hline JB486 & CGACTAGAGTCGACCTGCAGCCAAGCTTAGTAAAGCCCTC \\
\hline MV26 & ACGGCTGACATGGGAATTGC \\
\hline MV143 & CCTCTTACGTGCCGATCAACGTCTC \\
\hline MV145 & GCTGGTGATTCCGCTTTGCGACTCAACCTTTTCACCTAAAGTGCACCGACCGTGA \\
\hline MV146 & ACATCAGCGATCACCTGGCAGAC \\
\hline attBHKwtERI & AATTCCGCTTTGCGACTCAACCTTITTCACCTAAAGTGCACCGACCGTGAATG \\
\hline attBHKwtREV & GATCCATTCACGGTCGGTGCACTTAAGGTGAAAAAGGTTGAGTCGCAAAGCGG \\
\hline attBHKmutERI & AATTCCGCTTTGCGACACAACCTTTTTCACCTAAAGTGCACCGACCGTGAATG \\
\hline attBHKmutREV & GATCCATTCACGGTCGGTGCACTTTAGGTGAAAAAGGTTGTGTCGCAAAGCGG \\
\hline 40wtERI & AATTCTGCGACTCAACCTITTCACCTAAAGTGCACCG \\
\hline 40wtREV & GATCCCGGTGCACTTIAGGTGAAAAAGGTTGAGTCGCAG \\
\hline 40attBHKmutERI & AATTCTGCGACACAACCTTTTCACCTAAAGTGCACCG \\
\hline 40attBHKmutREV & GATCCCGGTGCACTTTAGGTGAAAAAGGTTGTGTCGCAG \\
\hline 30wtERI & AATTCTCAACCTIITICACCTAAAGTG \\
\hline 30wtREV & GATCCACTTTAGGTGAAAAAGGTTGAG \\
\hline 30attBHKmutERI & AATTCACAACCTTTTTCACCTAAAGTG \\
\hline 30attBHKmutREV & GATCCACTTTAGGTGAAAAAGGTTGTG \\
\hline 30attBHKamp2ORF1min & TाTGCTCACAACCTTTTCACCTAAAGTGGCACCCAGAAACGCTGGTGAAAGTAAAAGATGCTGAAGATCAGTT \\
\hline 30attBHKamp1ORF1min & CTTCACCAGCGTTTCTGGGTGCCACTTIAGGTGAAAAAGGTTGTGAGCAAAAACAGGAAGGCAAAATGCCGC \\
\hline 30attBHKamp2ORF2min & TाTGCTACACAACCTITTCACCTAAAGTGCACCCAGAAACGCTGGTGAAAGTAAAAGATGCTGAAGATCAGTT \\
\hline 30attBHKamp1ORF2min & CTTCACCAGCGTTTCTGGGTGCACTTAAGGTGAAAAAGGTTGTGTAGCAAAAACAGGAAGGCAAAATGCCGC \\
\hline 30attBHKamp2ORF3min & TTGGCTGCCACTTTAGGTGAAAAAGGTTGTCACCCAGAAACGCTGGTGAAAGTAAAAGATGCTGAAGATCAGTT \\
\hline 30attBHKamp1ORF3min & CTTTCACCAGCGTTTCTGGGTGACAACCTTTTCACCTAAAGTGGCAGCAAAAACAGGAAGGCAAAATGCCGC \\
\hline phiC31 ORF1 F & TाTGCTTGCGGGTGCCAGGGCGTGCCCTTGGGCTCCCCGGGCGCGTACTCCCACCCAGAAACGCTGGTGAAAG \\
\hline phiC31 ORF2 F & TTGCTGCGGGTGCCAGGGCGTGCCCTTGGGCTCCCCGGGCGCGTACTCCCCACCCAGAAACGCTGGTGAAAG \\
\hline phiC31 ORF3 F & TTGCTCGGGTGCCAGGGCGTGCCCTTGGGCTCCCCGGGCGCGTACTCCCCCACCCAGAAACGCTGGTGAAAG \\
\hline phiC31 ORF4 F & TाTGCTGGAGTACGCGCCCGGGGAGCCCAAGGGCACGCCCTGGCACCCGCACACCCAGAAACGCTGGTGAAAG \\
\hline phiC31 ORF5 F & TTTGCTGGGAGTACGCGCCCGGGGAGCCCAAGGGCACGCCCTGGCACCCGCCACCCAGAAACGCTGGTGAAAG \\
\hline phiC31 ORF6 F & TTTGCTGGGGAGTACGCGCCCGGGGAGCCCAAGGGCACGCCCTGGCACCCGCACCCAGAAACGCTGGTGAAAG \\
\hline phiC31 ORF1 R & CTTCACCAGCGTTTCTGGGTGGGAGTACGCGCCCGGGGAGCCCAAGGGCACGCCCTGGCACCCGCAAGCAAAAACAGGAAGGCAAAATG \\
\hline phiC31 ORF2 R & CTTCACCAGCGTTTCTGGGTGGGGAGTACGCGCCCGGGGAGCCCAAGGGCACGCCCTGGCACCCGCAGCAAAAACAGGAAGGCAAAATG \\
\hline phiC31 ORF3 R & CTTCACCAGCGTTCTGGGTGGGGGAGTACGCGCCCGGGGAGCCCAAGGGCACGCCCTGGCACCCGAGCAAAAACAGGAAGGCAAAATG \\
\hline phiC31 ORF4 R & CTTCACCAGCGTTCTGGGTGTGCGGGTGCCAGGGCGTGCCCTTGGGCTCCCCGGGCGCGTACTCCAGCAAAAACAGGAAGGCAAAATG \\
\hline phiC31 ORF5 R & CTICACCAGCGTTCTGGGTGGCGGGTGCCAGGGCGTGCCCTTGGGCTCCCCGGGCGCGTACTCCCAGCAAAAACAGGAAGGCAAAATG \\
\hline phiC31 ORF6 R & CTTCACCAGCGTTCTGGGTGCGGGTGCCAGGGCGTGCCCTTGGGCTCCCCGGGCGCGTACTCCCCAGCAAAAACAGGAAGGCAAAATG \\
\hline
\end{tabular}


containing an attP plasmid and a helper plasmid expressing the appropriate integrase gene under control of the temperature-sensitive CI857 promoter. Prior to conjugation, strains were diluted 1/100 from an overnight starter culture and grown to $\mathrm{OD}_{600}=0.3$. Conjugations were performed by two techniques: for the attB $\mathrm{HK}_{\mathrm{WT} / \mathrm{MUT}}$ strains, $0.5 \mathrm{ml}$ of donor was mixed with $4.5 \mathrm{ml}$ of recipient and applied to a $0.45 \mu \mathrm{m}$ filter (Millipore) by vacuum-filtration through a glass column. The attB ORF insertions into bla were performed by mixing $0.2 \mathrm{ml}$ of donor with $1.8 \mathrm{ml}$ of recipient, and following centrifugation at 6000 RPM for $5 \mathrm{~min}, \sim 1.8 \mathrm{ml}$ of supernatant was removed, the pellet resuspended in the remaining liquid media, and similarly placed onto a $0.45 \mu \mathrm{m}$ filter. For both techniques, the filters were then incubated on an LB-DAP plate for approx. $16 \mathrm{~h}$ prior to resuspension and plating. Recombinants were recovered by selecting for $\mathrm{Cm}$ resistance in DAP-free media, and recombination frequencies were measured as the ratio of recovered recombinants over donor CFUs. Each att site was tested three times.

\section{Minimum inhibitory concentration (MIC)}

The MICs of $E$. coli strains containing plasmids with either $a t t B$ inserted into bla, or bla fragments associated with attL and attR were performed by plating and aspirating $2 \mathrm{ml}$ of a 1/100 dilution of an overnight culture onto an LB/DAP agar petri dish. An Etest (bioMérieux) ampicillin antibiotic strip was placed onto the plate and incubated overnight at $37^{\circ} \mathrm{C}$, and the level of antibiotic resistance was scored the following day.

\section{Data analysis}

Recombination frequencies were analyzed for statistical significance using MATLAB software (The MathWorks, Inc., Natick, MA). 1 and 2-way analysis of variance (ANOVA) tests were performed using the anoval and anova2 functions. Tukey-Kramer post-hoc tests were performed using the multcompare function.

\section{DNA folding and protein structure analysis}

Secondary DNA structures were analyzed using the mfold software [46]. Protein residue charges were calculated by counting negatively charged residues Asp and Glu as -1 , and positively charged His, Lys, and Arg as +1 .

\section{Abbreviations}

Bla: $\beta$-lactamase; HK022: HK; Int: Integrase; MIC: Minimum inhibitory concentration; O region: Overlap region; ORF: Open reading frame; RDF: Recombination directionality factor; S-rec: Serine recombinase; SSR: Site-specific recombinase; Y-rec: Tyrosine recombinase

\section{Acknowledgments}

The authors thank Sean Colloms (Institute of Molecular Cell and Systems Biology, University of Glasgow, Glasgow, Scotland, UK) for providing $\Phi C 31$ plasmids. The authors thank Aleksandra Nivina and Jessica Bryant for critical reading of the manuscript.

\section{Funding}

Work in the Mazel laboratory is funded by the Institut Pasteur, the Institut National de la Santé et de la Recherche Médicale (INSERM), the Centre National de la Recherche Scientifique (CNRS-UMR 3525), the French National Research Agency (ANR-14-CE10-0007), the French Government's Investissement d'Avenir program, Laboratoire d'Excellence "Integrative Biology of Emerging Infectious Diseases" (grant n ANR-10-LABX-62-IBEID) and the European Union Seventh Framework Programme (FP7-HEALTH-2011-single-stage) "Evolution and Transfer of Antibiotic Resistance" (EvoTAR). MJB was supported by the Pasteur-Paris University (PPU) International PhD program.

\section{Availability of data and materials}

Raw data are available from the corresponding author on request.

\section{Authors' contributions}

MJB, MEV, and DM conceived and designed the experiments; MJB and MDG carried out the experiments; MJB, MDG, MEV, and DM analyzed the data; MJB and DM wrote the paper. All authors read and approved the final version of the manuscript.

Ethics approval and consent to participate

Not applicable.

\section{Consent for publication}

Not applicable.

\section{Competing interests}

The authors declare that they have no competing interests.

\section{Publisher's Note}

Springer Nature remains neutral with regard to jurisdictional claims in published maps and institutional affiliations.

Received: 14 April 2017 Accepted: 9 July 2017

Published online: 14 July 2017

\section{References}

1. Olorunniji FJ, Rosser SJ, Stark WM. Site-specific recombinases: molecular machines for the genetic revolution. Biochem J. 2016;473(6):673-84.

2. Grindley ND, Whiteson KL, Rice PA. Mechanisms of site-specific recombination. Annu Rev Biochem. 2006;75:567-605.

3. Thyagarajan B, Olivares EC, Hollis RP, Ginsburg DS, Calos MP. Site-specific genomic integration in mammalian cells mediated by phage phiC31 integrase. Mol Cell Biol. 2001;21(12):3926-34

4. Bischof J, Maeda RK, Hediger M, Karch F, Basler K. An optimized transgenesis system for drosophila using germ-line-specific phic31 integrases. Proc Natl Acad Sci U S A. 2007;104(9):3312-7.

5. Hirano N, Muroi T, Takahashi H, Haruki M. Site-specific recombinases as tools for heterologous gene integration. Appl Microbiol Biotechnol. 2011;92(2):227-39.

6. Khaleel T, Younger E, McEwan AR, Varghese AS, Smith MC. A phage protein that binds phiC31 integrase to switch its directionality. Mol Microbiol. 2011; 80(6):1450-63.

7. Petersen KV, Martinussen J, Jensen PR, Solem C. Repetitive, marker-free, site-specific integration as a novel tool for multiple chromosomal integration of DNA. Appl Environ Microbiol. 2013;79(12):3563-9.

8. Yagil E, Dolev S, Oberto J, Kislev N, Ramaiah N, Weisberg RA. Determinants of site-specific recombination in the lambdoid coliphage HK022. An evolutionary change in specificity. J Mol Biol. 1989;207(4):695-717.

9. Azaro MA, Landy A. In: Craig RC NL, Gellert M, Lambowitz AM, editors. Mobile DNA II. Washington, DC: ASM Press; 2002. p. 118-48.

10. Weisberg RA, Gottesmann ME, Hendrix RW, Little JW. Family values in the age of genomics: comparative analyses of temperate bacteriophage HK022. Annu Rev Genet. 1999:33:565-602.

11. Groth AC, Calos MP. Phage integrases: biology and applications. J Mol Biol. 2004;335(3):667-78.

12. Smith MC, Brown WR, McEwan AR, Rowley PA. Site-specific recombination by phiC31 integrase and other large serine recombinases. Biochem Soc Trans. 2010;38(2):388-94.

13. Tungsuchat T, Kuroda H, Narangajavana J, Maliga P. Gene activation in plastids by the CRE site-specific recombinase. Plant Mol Biol. 2006;61(4-5):711-8. 
14. Nakano M, Odaka K, Ishimura M, Kondo S, Tachikawa N, Chiba J, Kanegae Y, Saito I. Efficient gene activation in cultured mammalian cells mediated by FLP recombinase-expressing recombinant adenovirus. Nucleic Acids Res. 2001;29(7):E40

15. Qureshi SA. Beta-lactamase: an ideal reporter system for monitoring gene expression in live eukaryotic cells. BioTechniques. 2007;42(1):91-6.

16. Collinet B, Herve M, Pecorari F, Minard P, Eder O, Desmadril M. Functionally accepted insertions of proteins within protein domains. J Biol Chem. 2000; 275(23):17428-33.

17. Vandevenne M, Filee P, Scarafone N, Cloes B, Gaspard G, Yilmaz N, Dumoulin M, Francois JM, Frere JM, Galleni M. The Bacillus licheniformis BlaP beta-lactamase as a model protein scaffold to study the insertion of protein fragments. Protein Sci. 2007:16(10):2260-71.

18. Gersbach CA, Gaj T, Gordley RM, Barbas CF, 3rd. Directed evolution of recombinase specificity by split gene reassembly. Nucleic Acids Res 2010; 38(12):4198-206

19. Kadonaga JT, Gautier AE, Straus DR, Charles AD, Edge MD, Knowles JR. The role of the beta-lactamase signal sequence in the secretion of proteins by Escherichia coli. J Biol Chem. 1984;259(4):2149-54.

20. Itoh Y, Kanoh K, Nakamura K, Takase K, Yamane K. Artificial insertion of peptides between signal peptide and mature protein: effect on secretion and processing of hybrid thermostable alpha-amylases in Bacillus Subtilis and Escherichia coli cells. J Gen Microbiol. 1990;136(8):1551-8.

21. Valens M, Penaud S, Rossignol M, Cornet F, Boccard F. Macrodomain organization of the Escherichia coli chromosome. EMBO J. 2004;23(21):4330-41.

22. Thiel A, Valens M, Vallet-Gely I, Espeli O, Boccard F. Long-range chromosome organization in E. coli: a site-specific system isolates the Ter macrodomain. PLoS Genet. 2012:8(4):e1002672

23. Val ME, Skovgaard O, Ducos-Galand M, Bland MJ, Mazel D. Genome engineering in Vibrio cholerae: a feasible approach to address biological issues. PLoS Genet. 2012;8(1):e1002472.

24. Soler-Bistue A, Mondotte JA, Bland MJ, Val ME, Saleh MC, Mazel D. Genomic location of the major ribosomal protein gene locus determines Vibrio cholerae global growth and infectivity. PLoS Genet. 2015;11(4):e1005156.

25. Val ME, Marbouty M, de Lemos MF, Kennedy SP, Kemble H, Bland MJ, Possoz C, Koszul R, Skovgaard O, Mazel D. A checkpoint control orchestrates the replication of the two chromosomes of Vibrio cholerae. Sci Adv. 2016; 2(4):e1501914.

26. Sutcliffe JG. Nucleotide sequence of the ampicillin resistance gene of Escherichia coli plasmid pBR322. Proc Natl Acad Sci U S A. 1978;75(8):3737-41.

27. Kolot M, Malchin N, Elias A, Gritsenko N, Yagil E. Site promiscuity of coliphage HK022 integrase as tool for gene therapy. Gene Ther. 2015;22(7):602.

28. Nagaraja R, Weisberg RA. Specificity determinants in the attachment sites of bacteriophages HK022 and lambda. J Bacteriol. 1990;172(11):6540-50.

29. Demarre G, Guerout AM, Matsumoto-Mashimo C, Rowe-Magnus DA Marliere P, Mazel D. A new family of mobilizable suicide plasmids based on broad host range R388 plasmid (IncW) and RP4 plasmid (IncPalpha) conjugative machineries and their cognate Escherichia coli host strains. Res Microbiol. 2005;156(2):245-55

30. Rossignol M, Moulin L, Boccard F. Phage HK022-based integrative vectors for the insertion of genes in the chromosome of multiply marked Escherichia coli strains. FEMS Microbiol Lett. 2002;213(1):45-9.

31. Herrero M, de Lorenzo $\mathrm{V}$, Timmis KN. Transposon vectors containing nonantibiotic resistance selection markers for cloning and stable chromosomal insertion of foreign genes in gram-negative bacteria. J Bacteriol. 1990; 172(11):6557-67.

32. Groth AC, Olivares EC, Thyagarajan B, Calos MP. A phage integrase directs efficient site-specific integration in human cells. Proc Natl Acad Sci U S A. 2000;97(11):5995-6000

33. Brown WR, Lee NC, Xu Z, Smith MC. Serine recombinases as tools for genome engineering. Methods. 2011;53(4):372-9.

34. St-Pierre F, Cui L, Priest DG, Endy D, Dodd IB, Shearwin KE. One-step cloning and chromosomal integration of DNA. ACS Synth Biol. 2013;2(9):537-41.

35. Pradel N, Delmas J, Wu LF, Santini CL, Bonnet R. Sec- and tat-dependent translocation of beta-lactamases across the Escherichia coli inner membrane. Antimicrob Agents Chemother. 2009;53(1):242-8.

36. Li P, Beckwith J, Inouye $\mathrm{H}$. Alteration of the amino terminus of the mature sequence of a periplasmic protein can severely affect protein export in Escherichia coli. Proc Natl Acad Sci U S A. 1988;85(20):7685-9.

37. Pluckthun A, Knowles JR. The consequences of stepwise deletions from the signal-processing site of beta-lactamase. J Biol Chem. 1987;262(9):3951-7.
38. Barkocy-Gallagher GA, Bassford PJ Jr. Synthesis of precursor maltose-binding protein with proline in the +1 position of the cleavage site interferes with the activity of Escherichia coli signal peptidase I in vivo. J Biol Chem. 1992; 267(2):1231-8

39. Choo KH, Tan TW, Ranganathan S. SPdb-a signal peptide database. BMC Bioinf. 2005;6:249.

40. Choo KH, Ranganathan S. Flanking signal and mature peptide residues influence signal peptide cleavage. BMC Bioinf. 2008;(9, Suppl 12):S15.

41. Zacchi P, Sblattero D, Florian F, Marzari R, Bradbury AR. Selecting open reading frames from DNA. Genome Res. 2003;13(5):980-90.

42. Seehaus T, Breitling F, Dubel S, Klewinghaus I, Little M. A vector for the removal of deletion mutants from antibody libraries. Gene. 1992;114(2):235-7

43. Colloms SD, Merrick CA, Olorunniji FJ, Stark WM, Smith MC, Osbourn A Keasling JD, Rosser SJ. Rapid metabolic pathway assembly and modification using serine integrase site-specific recombination. Nucleic Acids Res. 2014; 42(4):e23

44. Duportet X, Wroblewska L, Guye P, Li Y, Eyquem J, Rieders J, Rimchala T, Batt $G$, Weiss R. A platform for rapid prototyping of synthetic gene networks in mammalian cells. Nucleic Acids Res. 2014;42(21):13440-51.

45. Gibson DG, Young L, Chuang RY, Venter JC, Hutchison CA 3rd, Smith HO. Enzymatic assembly of DNA molecules up to several hundred kilobases. Nat Methods. 2009;6(5):343-5.

46. Zuker M. Mfold web server for nucleic acid folding and hybridization prediction. Nucleic Acids Res. 2003;31(13):3406-15.

47. Ferrieres L, Hemery G, Nham T, Guerout AM, Mazel D, Beloin C, Ghigo JM. Silent mischief: bacteriophage mu insertions contaminate products of Escherichia coli random mutagenesis performed using suicidal transposon delivery plasmids mobilized by broad-host-range RP4 conjugative machinery. J Bacteriol. 2010;192(24):6418-27.

48. Biskri L, Bouvier M, Guerout AM, Boisnard S, Mazel D. Comparative study of class 1 integron and Vibrio cholerae superintegron integrase activities. J Bacteriol. 2005;187(5):1740-50.

49. Cohen SN, Chang AC. Revised interpretation of the origin of the pSC101 plasmid. J Bacteriol. 1977;132(2):734-7.

50. Yanisch-Perron C, Vieira J, Messing J. Improved M13 phage cloning vectors and host strains: nucleotide sequences of the M13mp18 and pUC19 vectors. Gene. 1985:33(1):103-19.

51. Guzman LM, Belin D, Carson MJ, Beckwith J. Tight regulation, modulation, and high-level expression by vectors containing the arabinose PBAD promoter. J Bacteriol. 1995:177(14):4121-30.

\section{Submit your next manuscript to BioMed Central and we will help you at every step:}

- We accept pre-submission inquiries

- Our selector tool helps you to find the most relevant journal

- We provide round the clock customer support

- Convenient online submission

- Thorough peer review

- Inclusion in PubMed and all major indexing services

- Maximum visibility for your research

Submit your manuscript at www.biomedcentral.com/submit 